\title{
Traduire
}

Revue française de la traduction

\section{Adaptation audiovisuelle : les réalités d'un secteur en crise}

Sylvestre Meininger

\section{(2) OpenEdition}

Journals

Édition électronique

URL : http://journals.openedition.org/traduire/973

DOI : $10.4000 /$ traduire.973

ISSN : 2272-9992

\section{Éditeur}

Société française des traducteurs

\section{Édition imprimée}

Date de publication : 1 mars 2008

Pagination : 16-23

ISSN : 0395-773X

\section{Référence électronique}

Sylvestre Meininger, «Adaptation audiovisuelle : les réalités d'un secteur en crise », Traduire [En ligne], 216 | 2008, mis en ligne le 01 mars 2008, consulté le 19 avril 2019. URL : http:// journals.openedition.org/traduire/973 ; DOI : 10.4000/traduire.973 


\title{
Adaptation audiovisuelle : les réalités d'un secteur en crise
}

\author{
SyLVESTRE MeININGER
}

Pour le grand public ou les étudiants en langue, l'adaptation audiovisuelle (doublage, sous-titrage, voice-over) apparaît souvent comme le domaine idéal où exercer le métier de traducteur.

La traduction pour le cinéma, la télévision ou l'édition vidéo promet la possibilité de travailler sur des films, des documentaires ou des séries. Traduire pour le cinéma, c'est permettre au public français de découvrir le nouveau prodige coréen ou chinois, de vibrer au rythme des films de Spielberg ou de Scorsese, ou d'explorer les univers de Lynch, Kitano, Burton ou Cronenberg. Traduire pour la télévision, c'est doubler ou sous-titrer des chefs-d'œuvre en noir et blanc, de passionnantes séries comme $\grave{A}$ la Maison-Blanche, Les Experts ou Urgences, ou des dessins animés destinés à la jeunesse ; c'est aussi adapter des documentaires qui abordent une impressionnante variété de sujets.

Les perspectives d'avenir au sein de cette profession peuvent également sembler encourageantes. La mondialisation permet aux œuvres audiovisuelles de circuler de plus en plus librement, les chaînes se multiplient et de nouveaux modes de diffusion apparaissent (vidéo à la demande, télévision numérique terrestre, vidéo sur téléphone mobile, etc.). Autant d'indices qui laissent à penser que ce secteur d'activité offre des débouchés.

En tant qu'association professionnelle, dont les membres connaissent bien leur secteur d'activité, l'ATAA (Association des Traducteurs Adaptateurs de l'Audiovisuel) se doit d'apporter une touche de réalisme à ce tableau idyllique. 


\section{1) Le statut d'auteur est le plus précaire qui soit}

Voilà une évidence qu'il n'est pas inutile de rappeler. Ayant le statut d'auteur, les traducteurs n'ont pas de contrat de travail, ils ne bénéficient d'aucune assurance-chômage, ils n'ont droit ni aux congés payés, ni aux congés spectacles et leur retraite ne peut dépasser la moitié du plafond de la Sécurité sociale.

Après une période d'activité, un intermittent du spectacle ou une personne travaillant en CDD peuvent toucher une indemnité s'ils ne retrouvent pas tout de suite du travail. Un traducteur aura beau travailler six mois ou un an d'affilée, le jour où il s'arrête, il n'a droit à rien : ni indemnités de chômage, ni congés payés. Il est donc parfois difficile de refuser du travail impliquant des délais trop courts ou des tarifs non rentables. Mais il ne faut pas oublier que lorsqu'on a accepté ces mauvaises conditions de travail, le client n'a aucune raison d'en proposer de meilleures par la suite.

\section{2) La prépondérance du relationnel}

Comme tous les métiers liés à l'audiovisuel, l'adaptation est avant tout une affaire de relations et de réseaux. Bien sûr, la qualité du travail fourni est prise en compte par les clients, mais elle ne suffit pas à les fidéliser ni à progresser dans le métier. Un traducteur ne peut espérer vivre de ce métier s'il reste chez lui à attendre que les clients l'appellent. Pour percer, ou même se maintenir, il faut savoir se rappeler au souvenir des commanditaires, entreprendre les démarches qui permettront d'en trouver de nouveaux, savoir se mettre en valeur et surtout savoir se faire respecter, soi et son travail, face à des interlocuteurs qui n'accordent souvent que peu de valeur aux activités intellectuelles et littéraires. Le démarchage, la négociation et les rapports de force font partie intégrante de ce métier.

\section{3) La nature des programmes}

On peut toujours imaginer qu'à force de persévérance, un auteur de doublage ou de sous-titrage qui aura fait preuve de son talent puisse 
un jour avoir la chance de travailler pour le cinéma. Mais auparavant, comme les traducteurs d'autres secteurs, il aura dû faire ses armes sur des textes nettement plus ingrats. En doublage, le soap ou l'animation sont aujourd'hui un passage obligé. En voice-over, c'est la télé-réalité qui domine et pour le sous-titrage, le travail pour le DVD. Or ces programmes sont rarement plus faciles à traduire que les œuvres plus intéressantes, mais ils sont trois, cinq ou dix fois moins bien payés. Et si on peut penser qu'il est normal de commencer "à la dure ", il ne faut pas oublier que de telles conditions de travail, couplées au caractère extrêmement répétitif de ces programmes, tendent à émousser le talent du traducteur et à lui donner de mauvaises habitudes (recherches bâclées, relectures insuffisantes, style appauvri, etc.).

Après plusieurs années, il est parfois possible de travailler sur des séries, des films télédiffusés ou des documentaires plus évolués, mais seulement si l'on a su se constituer un réseau et surtout se faire respecter en n'acceptant pas n'importe quel travail à n'importe quel tarif. Aujourd'hui, les cinq ans d'études nécessaires à l'obtention d'un diplôme de traducteur audiovisuel n'offrent aucune garantie quant à la qualité des conditions de travail et au niveau de rémunération. Bien au contraire, les jeunes traducteurs se voient souvent obligés de travailler énormément, avec des délais trop courts et pour des tarifs proches du SMIC horaire.

\section{4) Les réalités du marché : travailler plus pour gagner moins}

Dans les années 1980 , le sous-titre à destination de la vidéo était payé 15 francs. Aujourd'hui, 1,50 euro est considéré comme un bon tarif. Mais de plus en plus de traducteurs travaillent à 70 centimes ou 50 centimes le sous-titre. Une enquête de l'ATAA, réalisée auprès d'environ 150 traducteurs, établit la moyenne à environ un euro, soit une baisse de $60 \%$ en vingt ans. Jusqu'à récemment, le doublage destiné à la vidéo n'avait pas connu de véritable baisse. Mais notre enquête montre que l'apparition de logiciels de doublage virtuel a 
changé la donne. Aujourd'hui, il n'est pas rare de voir de jeunes traducteurs contraints de travailler à 150 ou même 100 euros la bobine, le tarif professionnel étant de 260 euros. Dans ce cas, la chute est encore plus spectaculaire : environ $-40 \%$ en deux ou trois ans.

Seul secteur où les traducteurs sont encore en contact avec les diffuseurs, le cinéma n'a pas subi de telles baisses. Mais les sorties en salle ne font vivre que $5 \%$ de la profession.

Comment en est-on arrivé là ? Il y a une quinzaine d'années, l'apparition des chaînes du câble puis du DVD a créé de réels débouchés pour l'adaptation audiovisuelle, notamment pour le sous-titrage. Mais cette augmentation du volume a eu deux effets pervers : l'effondrement des rémunérations et, plus grave encore, la création d'un déséquilibre durable entre l'offre et la demande d'adaptation. Cette situation est la cause principale de la crise que traverse notre profession, l'augmentation du volume de programmes à traduire au cours des années 1990 ayant attiré de nouveaux traducteurs qui se trouvent aujourd'hui trop nombreux, pour une quantité de travail qui s'est considérablement réduite.

En effet, la quasi-totalité des catalogues de programmes est désormais traduite et il est fort peu probable que la généralisation de la VOD ou de la TNT apportera un supplément de travail, les nouveaux diffuseurs pouvant, grâce au numérique, réutiliser les fichiers de sous-titres existants, que ceux-ci soient bons ou mauvais. Les versions doublées, du fait de leur prix de revient plus élevé que celui d'un soustitrage, sont rarement refaites. En 2005-2006, les ventes de DVD se sont écroulées de $30 \%$. La télévision par câble est tout aussi exsangue, comme le prouve le rachat de TPS par Canal+, concentration qui ne manquera pas de faire encore chuter les tarifs.

Un tel déséquilibre entre l'offre et la demande permet aux sociétés de postproduction spécialisées dans le doublage et/ou le sous-titrage d'exercer une pression constante sur les tarifs et les délais. En effet, depuis le boom des années 1990, ces sociétés sont venues se placer entre les diffuseurs et les traducteurs, réduisant ces derniers à une 
simple ligne " adaptation » dans les budgets globaux qu'elles proposent à leurs clients. En tant que prestataires techniques, elles n'ont la plupart du temps aucune compétence en traduction et n'accordent qu'une considération de façade à une activité qu'elles cherchent à rentabiliser autant que la duplication de master, l'incrustation ou l'encodage. Et quand le client demande une baisse du tarif global, ce qui arrive régulièrement, les sociétés de postproduction rognent sur le seul poste qui n'est pas protégé par un contrat de travail ou une convention collective : la traduction.

Ce mépris implicite est en partie lié au fait que la traduction, en plus d'être une activité littéraire, cumule une autre " tare ", celle d'être une profession très largement féminine. Or, on le sait depuis longtemps, qui dit profession féminine dit manque de reconnaissance et rémunérations inférieures. Ce constat mériterait un développement bien plus conséquent, et il touche certainement d'autres secteurs de la traduction, mais il est particulièrement vrai dans l'adaptation audiovisuelle. En effet, dans les entreprises à forte culture technique, et donc masculine, que sont les laboratoires, écrire des textes chez soi est souvent perçu comme une activité de loisir, qu'on peut exercer en gardant les enfants, et non comme un "vrai travail».

Ainsi, notre profession est probablement une des seules dont les tarifs ne suivent même pas l'inflation. Et lorsque le traducteur rappelle qu'il ne peut pas travailler à des tarifs cinq ou dix fois inférieurs au tarif professionnel, il n'est pas rare qu'il se voie cordialement informé que s'il n'est pas content, d'autres sont prêts à prendre sa place. À chacun de ne pas se laisser impressionner par ce genre de discours, qui en dit long sur l'importance que certaines sociétés accordent à la qualité.

Autre réalité de notre métier, conséquence directe de la mondialisation : les grandes multinationales de la traduction ont fait main basse sur une partie du marché de l'adaptation audiovisuelle. La France a été relativement épargnée jusqu'ici grâce à la détermination des professionnels du secteur et parce que la qualité de la langue a encore une importance dans notre pays. Cependant, ces multinationales sont déjà 
parvenues à détruire le métier dans les pays scandinaves, dans certains pays d'Europe de l'Est, en Angleterre, aux États-Unis et en Amérique Latine, régions où l'adaptation n'est plus un métier mais un job pour étudiants. Et il n'y a aucune raison de penser que ces sociétés ne vont pas continuer à essayer de s'implanter en France. Elles le font d'ailleurs déjà, en se basant à Londres ou à Bruxelles pour employer des traducteurs français qu'elles attirent grâce à des programmes intéressants, notamment les séries américaines, pour les faire travailler au dixième du tarif professionnel.

L'apparition de logiciels de doublage ou de sous-titrage représente un autre défi pour notre profession. En effet, si ces logiciels apportent des fonctionnalités utiles, ils permettent aussi de faire basculer sur le traducteur la charge financière liée aux investissements nécessaires à l'accomplissement du travail. En effet, grâce aux logiciels, les entreprises n'ont plus besoin de mobiliser les locaux et les machines nécessaires aux travaux techniques de repérage/détection ou simulation/ vérification. En d'autres termes, c'est désormais au traducteur de payer s'il veut travailler, en achetant un ordinateur récent, un logiciel et en assumant toutes les dépenses liées à son activité. Autre problème, d'ordre juridique : ces logiciels permettent aux clients d'imposer au traducteur d'effectuer les tâches techniques, comme le repérage en sous-titrage ou la détection en doublage. Et ce dernier se voit bien souvent imposé une rémunération en droits d'auteur. Or les règles de Sécurité sociale précisent que ces tâches doivent être payées en salaires, mode de rémunération plus avantageux pour le traducteur, mais plus cher pour l'employeur.

Et le problème va plus loin, certaines sociétés n'hésitant pas à démarcher les universités pour faire la publicité de leur logiciel de doublage ou de sous-titrage auprès des étudiants. Ceux qui se laissent éblouir par les belles promesses qu'on leur fait achètent un logiciel, dépense imprudente qui les obligera à accepter des tarifs dérisoires dans l'espoir de rentabiliser leur investissement. 
Le sous-titrage destiné aux personnes sourdes ou malentendantes offre-t-il de meilleurs débouchés ? L'obligation pour les chaînes réalisant plus de 2,5\% d'audience de faire sous-titrer leurs programmes pourrait le laisser penser. Une première précision s'impose : si ce métier est tout aussi utile que la traduction, il ne consiste pas à traduire des œuvres mais à adapter des programmes en langue française et des programmes étrangers déjà traduits en français. C'est pourquoi on parle souvent d'adaptation «du français vers le français ».

Quant aux débouchés que font miroiter certaines formations, ils se sont déjà considérablement réduits. En effet, confrontés à l'impossibilité de vivre de leur métier, de nombreux traducteurs audiovisuels se sont tournés vers le sous-titrage pour sourds et malentendants. Conséquence de l'augmentation du volume de travail : les tarifs ont déjà chuté d'environ $30 \%$ en quelques années. De plus, certaines sociétés spécialisées dans ce type de prestations obligent désormais les adaptateurs à accepter d'être payés en droits d'auteurs alors que d'après les règles de Sécurité sociale, ils devraient l'être en salaires d'intermittents.

\section{Conclusion}

Cet exposé peut paraître pessimiste. L'ATAA le considère comme réaliste. Aujourd'hui, la traduction audiovisuelle est un secteur saturé, à l'avenir incertain, où il est très difficile de se faire une place. Les personnes souhaitant choisir cette orientation doivent le faire en toute connaissance de cause : chaque année, les Masters d'adaptation audiovisuelle (anciennement appelés D.E.S.S.) envoient plusieurs dizaines d'étudiants vers un secteur pourtant sinistré, qui fait vivre à grand peine quelques centaines de personnes. De plus, pour des raisons qui nous paraissent difficiles à comprendre, d'autres universités envisagent d'ouvrir des formations supplémentaires. Puisque nous avons aujourd'hui la chance inestimable de vivre dans une économie de marché, il n'est pas inutile d'en rappeler une règle de base : lorsque l'offre d'un 22 bien ou d'un service dépasse la demande, le prix de ce bien ou de ce 
service se dévalue. L'effondrement de nos rémunérations en est la meilleure preuve.

Symptôme le plus grave de la saturation du secteur, on assiste depuis une dizaine d'années à une division très nette de la profession. D'un côté, quelques traducteurs vivant bien de leur métier, notamment ceux qui travaillent pour le cinéma. De l'autre, une majorité de traducteurs victimes de la sous-traitance et qui travaillent à la chaîne pour les laboratoires de doublage/sous-titrage dont les tarifs baissent régulièrement. Ceux qui sont prêts à travailler vite et beaucoup peuvent espérer gagner leur vie correctement, mais la chute continue des tarifs les entraîne souvent dans une spirale dangereuse, dont il est difficile de sortir. Et s'il peut être passionnant de se lancer à 25 ans dans la vie active en travaillant 50 heures par semaine, ça l'est nettement moins à 40 , surtout quand la rémunération n'a pas suivi.

Pour en savoir plus sur l'Association des traducteurs adaptateurs audiovisuels :

www.traducteurs-av.org

sylme@free.fr

Sylvestre Meininger est titulaire d'un doctorat en histoire du cinéma et traducteur audiovisuel (sous-titrage). Il traduit également pour des revues universitaires et pour le Monde diplomatique, tout en poursuivant son activité de recherche. 